\title{
ARTROSCOPIA DO QUADRIL EM ATLETAS
}

HIP ARTHROSCOPY IN ATHLETES

Giancarlo Cavalli Polesello',

Nelson Keiske Ono²,

Davi Gabriel Bellan³,

Emerson Kiyoshi Honda ${ }^{4}$,

Rodrigo Pereira Guimarães ${ }^{5}$,

Walter Riccioli Junior',

Guilherme Do Val Sella ${ }^{7}$

\section{RESUMO}

Objetivo: Confirmar a importância terapêutica da artroscopia do quadril em atletas cuja dor impede a função desportiva da articulação do quadril, sendo capaz de minimizá-la a ponto de ajudar o retorno à atividade esportiva em níveis satisfatórios. Métodos: Foram analisados 49 pacientes que praticam esporte (51 quadris), submetidos à artroscopia do quadril que apresentavam dor e incapacidade para a prática esportiva. $\mathrm{O}$ seguimento mínimo foi de 12 meses e o máximo de 74 meses (média de 39,0 meses). No período pré-operatório avaliou-se a localização da dor, sua intensidade segundo a Escala de Expressão Facial (EEF) e o grau de incapacidade utilizando-se o critério de Harris Hip Score modificado (HHS). Anotaram-se diferentes diagnósticos que levaram à indicação da artroscopia, como impacto femoroacetabular, lesão do lábio acetabular não secundária ao impacto femoroacetabular e outros. No período pós-operatório, os pacientes foram avaliados pelos mesmos métodos do período pré-operatório e pela análise subjetiva de retorno ao esporte. Resultados: Baseando-se no HHS e EEF pré e pós-operatórios, a análise estatística mostrou significância entre os valores. Observou-se alguma melhora em todos os casos e retorno ao esporte, de forma satisfatória, na maioria deles. Conclusão: Diante do que foi estudado, confirmamos que a artroscopia em atletas com lesões localizadas no quadril é técnica eficaz, capaz de promover o retorno à prática esportiva na maioria dos casos, sem dor e com função articular efetiva, desde que bem indicada.

Descritores - Artroscopia; Acetabular; Quadril; Esportes

\section{ABSTRACT}

Objective: To confirm the therapeutic importance of hip arthroplasty in athletes whose pain precludes sportive function of the hip joint, being able to minimize it to the extent of helping on the return of sports practice at satisfactory levels. Methods: 49 athlete patients (51 hips) submitted to hip arthroscopy complaining of pain and inability to practice sports were assessed. Follow-up time ranged from 12 to 74 months (mean: 39.0 months). Preoperatively, pain site, severity according to Facial Expression Scale (FES) and the degree of disability using the modified Harris Hip Score (HHS) were assessed. Different diagnoses were provided, which led to the indication of arthroscopy, such as femoralacetabular impact, acetabular lip injury not secondary to femoral-acetabular impact, etc. Postoperatively, the patients were assessed by using the same methods as used at baseline and by the subjective analysis of return to sports activities. Results: Based on pre-and postoperative HHS and FES, the statistical analysis showed significance between values. We found some improvement in all cases and return to sports activities at a satisfactory level in most of the cases. Conclusion: As a result of our study, we confirm that arthroscopy in athletes with local hip injuries is an effective technique, able to promote the return to sports practice in most of the cases, without pain, and with an effective joint function, provided well indicated.

Keywords - Arthroscopy; Acetabular; Hip; Sports

1 - Professor Assistente e Doutor da FCMSCSP. Assistente do Grupo de Quadril do Departamento de Ortopedia e Traumatologia da FCMSCSP.

2 - Professor Assistente e Doutor da FCMSCSP. Chefe do grupo de Quadril do Departamento de Ortopedia e Traumatologia da FCMSCSP.

3 - Médico Residente do Departamento de Ortopedia de Traumatologia da FCMSCSP.

4 - Instrutor de Ensino e Doutor da FCMSCSP. Membro Sênior do Grupo de Quadril do Departamento de Ortopedia e Traumatologia da FCMSCSP.

5 - Instrutor de Ensino e Mestre da FCMSCSP. Assistente do grupo de Quadril do Departamento de Ortopedia e Traumatologia da FCMSCSP.

6 - Médico Ortopedista. Assistente do Grupo de Quadril do Departamento de Ortopedia e Traumatologia da FCMSCSP.

7 - Médico Ortopedista do Departamento de Ortopedia e Traumatologia da FCMSCSP.

Trabalho realizado no Departamento de Ortopedia e Traumatologia da Faculdade de Ciências Médicas da Santa Casa de São Paulo (FCMSCSP)

Correspondência: Av. Aicás, 491, apto 171 - CEP 04086-101 - São Paulo - SP - Brasil. E-mail: giancarlopolesello@hotmail.com

Declaração: Não houve auxílio, de qualquer espécie, a esta pesquisa e não há conflitos de interesse dos autores em relação ao presente manuscrito, conforme Resolução $\mathrm{n}^{\circ} 1.595 / 2000$ do Conselho Federal de Medicina. 


\section{INTRODUÇÃO}

A artroscopia do ombro e joelho em atletas é procedimento comum e suas indicações, bem definidas. Com o tempo, a artroscopia de outras articulações como cotovelo, tornozelo e punho foram se popularizando. No quadril esse entusiasmo foi retardado, pois é articulação profunda e seu acesso é mais difícil, pela própria anatomia articular. Mesmo assim, já é realidade tanto nos Estados Unidos da América e Europa como no Brasil e toda a América Latina, com indicações cada vez mais freqüentes, em diferentes afecções ${ }^{(1)}$.

Desde que Glick ${ }^{(2)}$ publicou a técnica com o paciente posicionado em decúbito lateral, após descobrir que se consegue visibilizar grande área articular pelo vetor lateral de tração, a artroscopia do quadril ganhou grande impulso, demonstrando-se procedimento seguro e de grande importância diagnóstica e terapêutica.

Com o crescente número de praticantes de esportes em busca da estética corporal e uma vida mais saudável, pretendendo aumentar seu desempenho, o número de afecções do quadril relacionadas ao esporte vem aumentando ${ }^{(3)}$.

A principal causa de dor no quadril em atletas é a lesão do lábio acetabular, tanto por causas traumáticas agudas quanto, muito mais freqüentemente, por mecanismos complexos não totalmente elucidados ${ }^{(4)}$. O impacto femoroacetabular (IFA) é uma dessas causas e vem ganhando importância como a principal causa de lesão do lábio acetabular.

Os procedimentos artroscópicos podem ajudar neste contexto, permitindo recuperação satisfatória a ponto de permitir o retorno ao esporte ${ }^{(5)}$.

O objetivo deste estudo é confirmar a importância terapêutica da artroscopia do quadril em atletas com dor incapacitante à prática esportiva.

\section{MÉTODOS}

Definimos prática esportiva o conjunto dos exercícios físicos praticados com regularidade, individual ou coletivamente $^{(6)}$. De acordo com este conceito foram avaliados 49 indivíduos praticantes de esporte (51 quadris), que apresentavam dor na articulação coxofemoral e incapacidade para a atividade esportiva. Destes, 32 eram do sexo masculino $(65,3 \%)$ e 17 do feminino (34,7\%). A idade variou de 16 a 51 anos (numa média de 33,1 anos). Entre os indivíduos avaliados, 42 eram de cor branca $(85,8 \%)$, três de cor negra $(6,1 \%)$, três de cor parda $(6,1 \%)$ e um de cor amarela $(2,0 \%)$. O quadril mais acometido foi o direito em 33 indivíduos $(64,7 \%) \mathrm{e}$ o esquerdo em $18(35,3 \%)$. O esporte mais praticado foi o futebol em 14 casos $(28,5 \%)$. O seguimento mínimo foi de 12 meses e tempo máximo 74 meses (numa média de 39 meses). No período pré-operatório, avaliou-se a localização da dor e sua intensidade segundo a escala visual de dor $(\mathrm{EEF})^{(7)}$ e o grau de incapacidade, utilizando-se o critério de Harris Hip Score modificado por Bird (HHS) ${ }^{(8)}$.

Após o procedimento cirúrgico, os pacientes foram reavaliados em períodos diversos, utilizando-se os mesmos critérios do período pré-operatório (HHS e EEF).

Para avaliar se houve diferença entre os valores de EEF antes e depois da cirurgia, foi realizado o Teste de Wilcoxon, utilizado para testar variáveis contínuas. Consideramos valores de $\mathrm{p}<0,05$ para significância estatística nas diferenças dos valores de EEF antes e após a artroscopia.

Para testar à variável HHS, o Teste de Wilcoxon foi utilizado considerando valores de $\mathrm{p}<0,05$ para significância estatística em relação à diferença de valores do HHS antes e após a artroscopia.

Para analisar os vários diagnósticos que provocaram a indicação cirúrgica, os pacientes foram divididos em três grupos segundo o diagnóstico intra-operatório denominados: grupo A, aqueles com diagnóstico de impacto femoroacetabular $(54,9 \%)$, grupo $\mathrm{B}$, aqueles com lesão do lábio acetabular não secundário ao IFA $(35,5 \%)$ e grupo C, pacientes com outros diagnósticos $(9,6 \%)$ (Tabelas 1 e 2). Todos foram submetidos á correção das deformidades anatômicas por osteoplastia, tanto acetabular (pincer) como femoral (came) quando presentes.

Os valores de HHS e EEF pré e pós-operatórios foram estudados utilizando-se o teste de Kruskal-Wallis,

Tabela 1 - Freqüência Diagnóstica dos Grupos A, B e C.

\begin{tabular}{l|c|c}
\hline Diagnóstico & Freqüência & Percentual \\
\hline Grupo A - Impacto fêmoro-acetabular & 28 & $54,9 \%$ \\
\hline $\begin{array}{l}\text { Grupo B - Lesão labial não secundária a impacto } \\
\text { fêmoro-acetabular }\end{array}$ & 18 & $35,5 \%$ \\
\hline Grupo C - Outros diagnósticos & 5 & $9,6 \%$ \\
\hline Total & 51 & $100,0 \%$ \\
\hline
\end{tabular}


Tabela 2 - Freqüência Diagnóstica do Grupo C

\begin{tabular}{l|c|c}
\hline Diagnóstico & Freqüência & Percentual \\
\hline Tendinite crônica do tendão do músculo ílio-psoas & 2 & $3,9 \%$ \\
\hline Ressalto da banda ílio tibial & 1 & $1,9 \%$ \\
\hline Lesão do ligamento redondo & 1 & $1,9 \%$ \\
\hline Doença inflamatória do quadril & 1 & $1,9 \%$ \\
\hline Total & 5 & $9,6 \%$ \\
\hline
\end{tabular}

com o intuito de verificarmos possíveis diferenças entre os três grupos estudados. Consideramos valores de $\mathrm{p}<0,05$ para significância estatística.

Para avaliarmos da forma mais simplificada possível o nível de retorno às atividades esportivas no pósoperatório, utilizamos critério subjetivo: se o paciente retornou ou não ao esporte. Para os que retornaram, foi perguntado qual o nível desse retorno: satisfatório ou insatisfatório. Foi considerado o retorno satisfatório aquele assintomático, com função normal.

\section{RESULTADOS}

Identificamos que 47 pacientes $(96,0 \%)$ retornaram de alguma forma ao esporte. Entre eles, $36(73,0 \%)$ com desempenho normal, assintomático, 11 (23,0\%) o fizeram de maneira considerada por eles insatisfatória e dois (4\%) abandonaram o esporte por apresentarem dor no quadril durante a sua prática (gráficos 1 e 2). Quando analisados todos os 51 casos, a variável HHS observada no período pré-operatório apresentou média de 62,3 pontos enquanto que no período pós-operatório a média

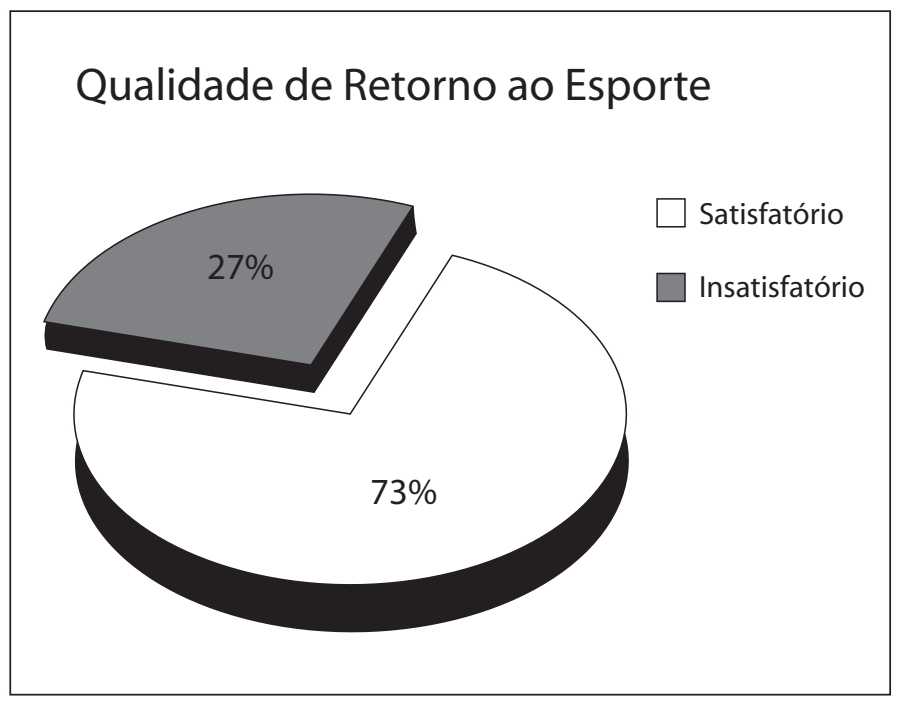

Gráfico 1 - Escala subjetiva de retorno ao esporte

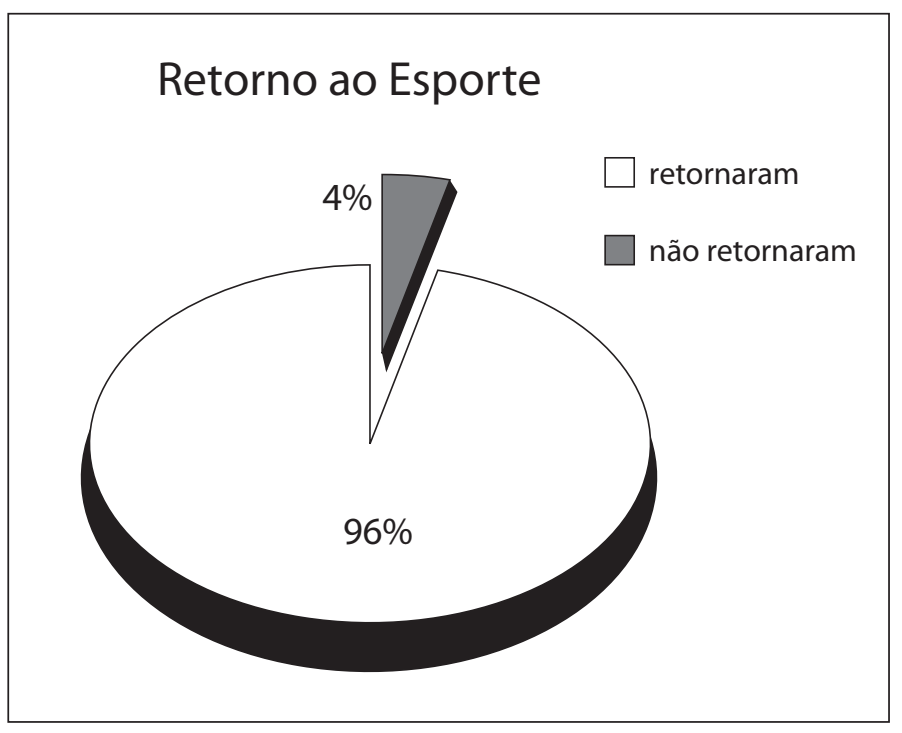

Gráfico 2 - Avaliação do retorno ao esporte

foi de 98,3 pontos, valor estatisticamente significante ( $p$ $<0,001)$. Quanto a EEF, que no período pré-operatório foi em média 2,16 pontos, no período pós-operatório foi 5,86 pontos, valor este estatisticamente significante $(\mathrm{p}<0,001)$.

Quando estudados os grupos separadamente observamos no grupo A variação de 62,2 pontos pré-operatórios na variável HHS para 98,7 pontos na avaliação pós-operatória ( $\mathrm{p}<0,001)$ e na variável EEF 2,2 pontos pré-operatórios para 5,9 pontos no pós-operatório ( $p<0,001)$. O grupo B apresentou melhora de 65,5 pontos para 99,1 no HHS ( $\mathrm{p}<0,001)$ e de 2,1 pontos para 5,9 na variável EEF ( $p<0,001$ ), enquanto o grupo C a variação foi de 54,6 pontos para 94,9 no HHS $(\mathrm{p}<0,028)$ e de 1,5 pontos para 5,3 pontos na variável $\operatorname{EEF}(p<0,027)$ (gráficos 3 e 4).

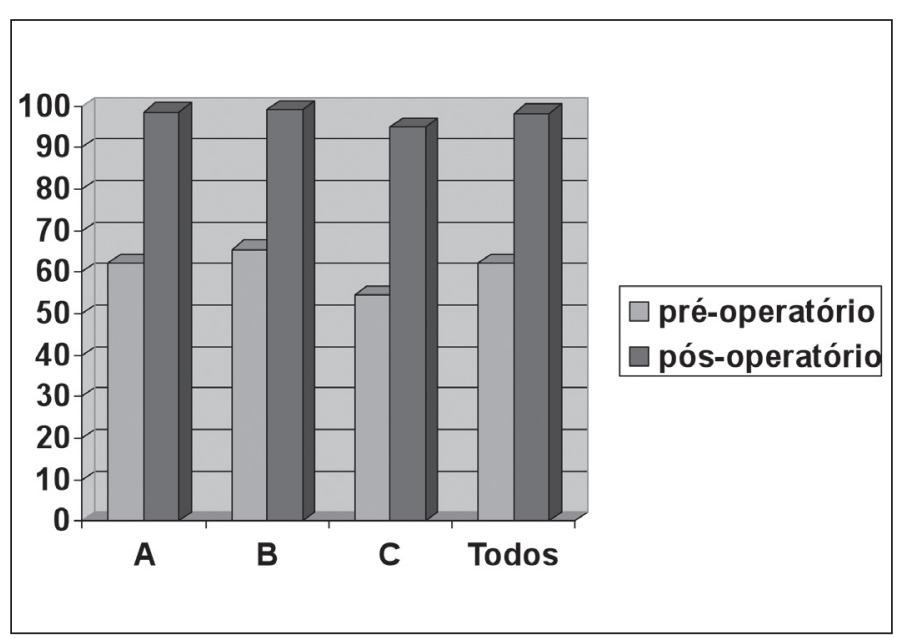

Gráfico 3 - Variação pré e pós-operatória da variável HHS 


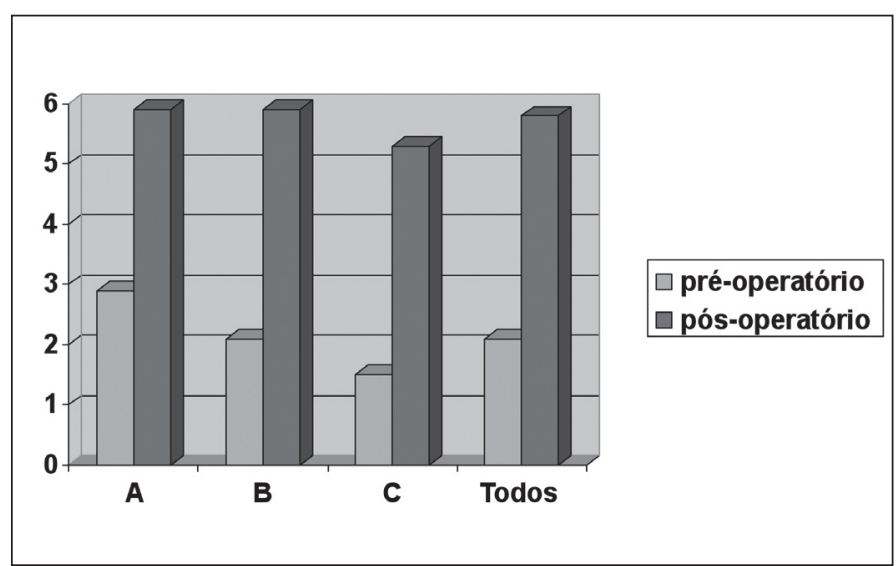

Gráfico 4 - Variação pré e pós-operatória da variável EEF

Quando comparamos a melhora clínica entre os grupos, isto é, se um grupo teve melhores resultados do que outro, não encontramos diferenças estatisticamente significativas, $\mathrm{p}<0,126$ para a variável HHS e $\mathrm{p}<0,918$ para EEF.

\section{DISCUSSÃO}

Na busca por saúde e qualidade de vida, a população cada vez mais procura no esporte a solução. O número de pessoas que praticam esporte regularmente aumenta a cada ano. Atletas amadores ou profissionais são submetidos a grandes esforços, que podem afetar suas articulações as tornando propícias às lesões ${ }^{(5)}$. Em conseqüência desta conduta esportiva os choques e traumas são cada vez mais freqüentes sobre as estruturas esqueléticas, aumentando o risco de contusões e lesões articulares. No mesmo sentido, a exigência cada vez maior da capacidade física aumenta o risco dessas lesões ${ }^{(9)}$. A evolução médico-tecnológica ocorrida nas últimas décadas teve impacto no esporte com importante avanço na preparação física dos atletas e conseqüente exigência de máximo desempenho ${ }^{(10)}$. O mecanismo da lesão pode ser devido a trauma direto ou a esforços repetitivos ${ }^{(5)}$. Quanto à topografia das lesões há maior acometimento dos membros inferiores, achado que é provavelmente explicado pelo fato de ser o futebol o esporte mais praticado no nosso meio $^{(10-12)}$.

Byrde e Ganz et al consideram a combinação da flexão, adução e rotação interna do quadril provocando dor na região inguinal (FADIR-test) como o teste mais sensível para a lesão intra articular do quadril ${ }^{(13,14)}$. McCarthy et $\mathrm{al}^{(15)}$ citam que a artroscopia do quadril é procedimento valioso e viável, e a indicam para pacientes com dor no quadril refratária ao tratamento clínico, com duração maior do que seis meses.

O impacto femoroacetabular vem ganhando importância como o principal causador da lesão do lábio acetabular. É definido como contato anormal entre o acetábulo e o colo femoral durante a mobilização do quadril, principalmente na flexão e rotação interna, limitando a amplitude de movimento ${ }^{(16)}$.

Londers et al analisaram 56 casos de artroscopias do quadril realizadas em pacientes com queixa de dor nesta articulação por mais do que seis meses, dor refratária ao tratamento clínico e com FADIR-test positivo ${ }^{(17)}$. Encontraram $80 \%$ de bons resultados com retorno as atividades da vida diária e esportivas; $2 \%$ dos pacientes referiram piora da dor e não puderam retornar as atividades esportivas, identificadam lesão do lábio acetabular em $62 \%$ dos casos e correlacionaram os maus resultados com o grau das lesões cartilaginosas presentes (Outerbridge III e IV ${ }^{(18)}$ ), porém não fizeram menção ao impacto femoroacetabular como possível causa da lesão do lábio e não descreveram seu tratamento nos procedimentos artroscópicos realizados, o que fizemos neste estudo. Londers et al demonstraram que em $11 \%$ a melhora foi temporária com o retorno dos sintomas prévios e em todos eles a lesão cartilaginosa era leve (Outerbridge I e II) ou ausente, o que sugere uma causa estrutural para o retorno dos sintomas ${ }^{(17)}$.

Heyworth et al revisaram 24 casos de artroscopia do quadril que evoluíram para maus resultados em um período médio de seis meses e observaram que 19 deles (79\%) apresentavam impacto femoroacetabular identificável nas radiografias pré-operatórias, porém não tratados durante o procedimento artroscópico ${ }^{(19)}$. Eles sugerem que as lesões do lábio acetabular podem apresentar alterações estruturais do quadril subdiagnosticadas e que o IFA não adequadamente tratado é a principal causa de persistência dos sintomas após desbridamento das lesões do lábio acetabular ${ }^{(19)}$.

Byrd et al observaram em 42 atletas (44 quadris), que 27 (61,3\%) apresentavam lesão do lábio acetabular, porém os autores não descreveram se eram lesões isoladas ou quais as possíveis causas ${ }^{(20)}$. $\mathrm{O}$ índice percentual foi discrepante ao observado em nossa casuística (18 casos - 35,5\%). Isto provavelmente se deve à separação dos casos de lesão labial decorrentes de impacto femoroacetabular dos casos secundários a outras afecções. 
Philippon registrou melhora no HHS de todos os seus atletas, e em sua casuística observou $84,8 \%$ de retorno ao esporte e $15,2 \%$ de incapacidade ao retorno esportivo prévio $^{(5)}$. Nossa casuística demonstrou que a maioria dos pacientes retornou ao esporte de alguma forma, $76 \%$ de maneira assintomática.

McCarthyem et al revendo 170 casos de artroscopia em quadris displásicos, encontraram em $42 \%$ deles lesão do lábio acetabular que apresentaram $85 \%$ de bons resultados após o desbridamento artroscópico ${ }^{(4)}$.

Em nosso estudo encontramos a lesão do lábio acetabular como a principal causa de dor dos quadris submetidos a artroscopia, dado este semelhante aos da literatura $^{(4)}$. Optamos por separar as lesões secundárias ao IFA para estabelecer a importância dessa afecção, muitas vezes subdiagnosticada e sugerida como a principal causa de persistência dos sintomas pós artroscopia $^{(19)}$. Tentamos encontrar diferenças entre os pacientes submetidos a sutura ou desbridamento das lesões, porém pelo tamanho da amostra e pelo tempo de seguimento isto não foi possível. Temos optado pela sutura em detrimento da ressecção da lesão baseados em trabalhos como Ikeda et al que compararam as lesões do lábio acetabular às lesões meniscais ${ }^{(21)}$. Ferguson et al citam como importantes as funções do lábio acetabular, que justificam a sua reparação: absorção de impacto, distribuidor de impacto, distribuidor de pressão e estabilizador e selador articular ${ }^{(22)}$. Outros autores já citaram que a sutura das lesões do lábio acetabular promovem melhores resultados que a ressecção do mesmo ${ }^{(23)}$. Não correlacionamos o grau de lesão da cartilagem com os resultados funcionais em nossos pacientes, pois em nenhum dos casos havia pacientes com perda do espaço articular, porém este parece ser um ponto de forte associação e deve ser levado em consideração $^{(17,19)}$. Encontramos $96 \%$ de retorno ao esporte pós artroscopia, sendo que $76 \%$ foram de forma satisfatória, ou seja, assintomática e semelhante ao período pré lesão. Esse dado é semelhante ao encontrado na literatura e evidencia o bom resultado da técnica artroscópica $^{(5,17)}$.

Os bons resultados do tratamento de qualquer afecção são sempre secundários a boa indicação do proce- dimento adotado e boa seleção dos pacientes. Sendo assim, pacientes portadores de artrose no quadril, radiograficamente evidenciada por perda do espaço articular e formação de osteófitos não são bons candidatos a qualquer procedimento artroscópico nessa articulação, sejam eles atletas ou não ${ }^{(13)}$.

Byrd et al acreditam que a melhora clínica é máxima aos três meses de seguimento pós operatório e que estes resultados tendem a ser melhores em pacientes mais jovens e tendem a permanecerem estáveis num período mínimo de cinco anos ${ }^{(20)}$.

A comparação direta entre todos os diagnósticos não foi possível devido ao pequeno número de casos de pacientes portadores das outras lesões, sendo necessário o agrupamento destes em um único grupo (cinco casos - 9,8\%). Desta forma, comparando-os, não observamos melhores resultados da técnica artroscópica entre os diferentes diagnósticos encontrados em nossos atletas, não sendo este um critério prognóstico de artroscopia.

O HHS é uma escala de avaliação imperfeita do quadril, porém é conhecida, padronizada, validada e o ortopedista está familiarizado com ela. Isso permite uma avaliação objetiva e confiável da dor e funcionalidade dessa articulação ${ }^{1 *}$. Não dispomos de uma escala qualitativa e padronizada que avalie especificamente o retorno ao esporte, como julgarmos importante a análise dos dados obtidos, utilizamos a escala subjetiva de retorno ao esporte. A maioria dos pacientes relataram retorno assintomático às atividades esportivas, o que reflete o bom resultado da técnica artroscópica neste grupo de atletas.

\section{CONCLUSÃO}

Diante do que foi estudado, confirmamos que a artroscopia em atletas com lesões localizadas no quadril é técnica eficaz, capaz de promover o retorno à prática esportiva, na maioria dos casos, com função articular efetiva e indolor.

\section{AGRADECIMENTO}

Agradecemos ao Núcleo de Apoio à Publicação o suporte técnico-científico à publicação deste manuscrito.

$1^{*}$ Polesello GC, Honda E, Ono N, Guimarães R, Aristide RSA. Artroscopia do Quadril - Atualização. Educação continuada/SBOT 25:32 Junho 2005. 


\section{REFERÊNCIAS}

1. Kelly BT, Williams RJ 3rd, Philippon MJ. Hip arthroscopy: current indications, treatment options, and management issues. Am J Sports Med 2003;31(6):1020-37.

2. Glick JM. Hip arthroscopy. The lateral approach. Clin Sports Med. 2001;20(4):733-47.

3. Raymundo JLP, Reckers LJ, Locks R, Silva L, Hallal PC. Perfil das lesões e evolução da capacidade física em atletas profissionais de futebol durante uma temporada. Rev Bras Ortop. 2005;40(6):341-48.

4. McCarthy JC, Lee JA. Hip arthroscopy: indications, outcomes, and complications. J Bone Joint Surg Am. 2005;87(5):1138-45.

5. Philippon MJ. Arthroscopy of the hip in the management of the athlete. In: McGinty JB. Operative arthroscopy. 3rd ed. Philadelphia: Lippincott, Williams \& Wilkins 2003, p.879-83.

6. Ferreira $\mathrm{ABH}$. Novo Dicionário da Língua Portuguesa. 2a ed. Rio de Janeiro: Nova Fronteira; 1986. p.708.

7. Pimenta CAM, Teixeira MJ. Avaliação da dor. Rev Med (São Paulo). 1997; 76(edição especial):27-35.

8. Byrd JW, Jones KS. Prospective analysis of hip arthroscopy with 2-year follow-up. Arthroscopy. 2000;16(6):578-87.

9. Bjordal JM, Arnly F, Hannestad B, Strand T. Epidemiology of anterior cruciate ligament injuries in soccer. Am J Sports Med. 1997;25(3):341-5.

10. Cohen M, Abdalla RJ, Ejnisman B, Amaro JT. Lesões ortopédicas no futebol. Rev Bras Ortop. 1997;32(11):940-4.

11. Pedrinelli A. Incidência de lesões traumáticas em atletas de futebol [dissertação]. São Paulo: Faculdade de Medicina da Universidade de São Paulo; 1994.

12. Ladeira CE. Incidência de lesões no futebol: um estudo prospectivo com jogadores masculinos adultos amadores canadenses. Rev Bras Fisioter. 1999;4(1):39-47.

13. Byrd JW. Hip arthroscopy: pacients assessment and indications. Instr Course Lect. 2003;52:711-19.

14. Ganz R, Parvizi J, Beck M, Leunig M, Notzli H, Siebenrock KA. Femoroacetabular impingement: a cause for osteoarthritis of the hip. Clin Orthop Relat Res. 2003;(417):112-20.

15. McCarthy JC, Busconi B. The role of hip arthroscopy in the diagnosis and treatment of hip disease. Orthopedics. 1995;18(8):753-6.

16. Tannast M, Siebenrock KA, Anderson SE. Femoroacetabular impingement: radiographic diagnosis--what the radiologist should know. AJR Am J Roentgenol. 2007;188(6):1540-52.

17. Londers J, Van Melkebeek J. Hip arthroscopy: outcome and patient satisfaction after 5 to 10 years. Acta Orthop Belg. 2007;73(4):478-83.

18. Outerbridge RE. The etiology of chondromalacia patellae. J Bone Joint Surg Br. 1961;43:752-7.

19. Heyworth BE, Shindle MK, Voos JE, Rudzki JR, Kelly BT. Radiologic and intraoperative findings in revision hip arthroscopy. Arthroscopy. 2007;23(12):1295-302.

20. Byrd JW, Jones KS. Hip arthroscopy in athletes. Clin Sports Med. 2001;20(4):749-61.

21. Ikeda T, Awaya G, Suzuki S, Okada Y, Tada H. Torn acetabular labrum in yung patients: arthroscopic diagnosis and management. J Bone Joint Surg Br. 1988;70(1):13-6.

22. Ferguson SJ, Bryant JT, Ganz R, Ito K. The influence of the acetabular labrum on hip joint cartilage consolidation: a poroelastic finite element model. J Biomech. 2000;33(8):953-60.

23. Espinosa N, Rothenfluh DA, Beck M, Ganz R, Leunig M. Treatment of femoro-acetabular impingement: preliminary results of labral refixation. J Bone Joint Surg Am. 2006;88(5):925-35. 